\title{
Combined electro-thermal model for PV panels
}

AKADÉMIAI KIADÓ

Pollack Periodica •

An International Journal

for Engineering and

Information Sciences

16 (2021) 1, 7-13

DOI:

$10.1556 / 606.2020 .00145$

(c) 2020 The Author(s)

\section{ORIGINAL RESEARCH} PAPER

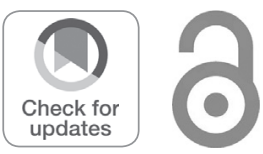

* Corresponding author.

E-mail: ali.k.abdulrazzaq@edu.bme.hu

\section{Ali Kareem Abdulrazzaq ${ }^{1,2 *} \oplus$, György Bognár ${ }^{1}$ and Balázs Plesz ${ }^{1}$}

${ }^{1}$ Department of Electron Devices, Faculty of Electrical Engineering and Informatics, Budapest University of Technology and Economics, Magyar tudósok körútja 2, H-1117, Budapest, Hungary

${ }^{2}$ Department of Electrical and Electronics Engineering, Thi-Qar University, P.O.B 0535, 64001, Nassiriya, Iraq

Received: December 31, 2019 • Revised manuscript received: April 27, 2020 - Accepted: May 20, 2020

Published online: March 11, 2021

\begin{abstract}
This paper presents a combined electro-thermal model to serve the aim of accurate output power prediction of photovoltaic systems, based on the concept of the thermal energy balance. The electrical sub-model is built based on fitting a surface to the current-voltage curves collected under wide range temperatures and irradiances. For this purpose, the current-voltage characteristic curves are reproduced using two different methods. The thermal sub-model considers all the effective heat transfer mechanisms to estimate the photovoltaic module junction temperature. The Newton-Raphson iterative method is used as a solving algorithm to calculate the photovoltaic junction temperature. The collected results prove the applicability of the model under a wide range of environmental conditions.
\end{abstract}

\section{KEYWORDS}

electro-thermal model, solar cells, current-voltage characteristics, heat loss mechanisms

\section{INTRODUCTION}

PV devices directly convert the received solar irradiance to electrical energy. However, the efficiency of a typical commercial PhotoVoltaic (PV) module is about $13-20 \%[1,2]$. The remaining of the received solar irradiance is converted to heat. The higher the temperature of a PV module, the lower is the efficiency [3-5]. Hence, this concept acts as a closed-loop relation. Besides the effect of the temperature on the efficiency, high temperature directly affects the PV module reliability and the long-term performance [6]. Therefore, it is evident that accurate power prediction and performance monitoring are more effective when both electrical and thermal sub-models are combined.

Many sources in the recent literature cover the combined electro-thermal modeling of PV systems. The rest of this section will summarize some of these research papers. In [7], the authors proposed an electro-thermal model based on the Single Diode Model (SDM) and thermal feedback circuit to model the temperature dependence of the electrical parameters by using an analytical approach aiming for reducing the computational effort. Another coupled electro-thermal model is proposed in [8] by including environmental conditions like temperature, irradiance, and wind velocity. The authors also use the SDM as a basement for building the electrical sub-model. To extract the SDM five parameters, they use an analytical model to solve the SDM equation at three characteristic points, i.e., short-current point, open-voltage point, and Maximum Power Point (MPP). They consider a linear dependence of the five parameters on temperature and irradiance to calculate their corresponding values. Their thermal sub-model is consisting of an equivalent thermal resistances circuit that is formulated using the energy conservation equation and solved using an iterative method. The authors in [9] proposed a combined model based on the energy balance of the solar module. For the electrical sub-model, they use the Lambert W function-based solution of the SDM. They proposed a specific formulation to determine the temperature and irradiance effects on the five parameters of the SDM. For the thermal sub-model, they use the Cauer RC ladder as 
a core for their model by incorporating additional RC components to represent the heat flow baths from the top and bottom of the PV module. Another coupled model was presented in [10]. Their electrical sub-model provides the SDM five parameter values at the reference conditions as a first step; then it scales these parameters to the selected environmental condition by a specific formulation with the help of additional two parameters. The other two parameters are calculated using two additional equations. They proposed a three-dimensional numerical thermal sub-model to predict the PV module thermal behavior.

The aim of the presented work is to propose a new method for estimating the PV module output power with the focus on enhancing the accuracy by combining both electrical and thermal sub-models. Hence, the instantaneous value of the PV junction temperature delivered by the thermal sub-model will act as an input to the electrical submodel to accurately calculate the electrical output. The output power, on the other hand, will be fed back to the thermal sub-model to be included in the thermal energy balance equation and recalculate the temperature. The results of the model will also provide information about the temperature profile across the PV module. The electrical sub-model is build using a simple and efficient method to ensure low computational cost while maintaining accurate results.

\section{ELECTRICAL SUB-MODEL}

In this work, the SDM has been used to build the electrical sub-model. The implicit non-linear mathematical representation of the SDM is given as $[11,12]$ :

$$
I=I_{p h}-I_{o}\left(e^{\frac{\left(V+I R_{s}\right)}{n V_{T}}}-1\right)-\frac{V+I R_{s}}{R_{s h}} .
$$

The five parameters that appear in the SDM model equation characterize the PV module at a specific environmental condition. These parameters are the photo-generated current $\left(I_{p h}\right)$, reverse saturation current $\left(I_{o}\right)$, the ideality factor of the PV cell $(n)$, cell series resistance $\left(R_{s}\right)$, and shunt resistance $\left(R_{s h}\right)$. For a panel consisting of multiple cells connected in series and/or in parallel, the number of these cells has to consider and can be given as [13]:

$$
I=N_{p} I_{p h}-N_{p} I_{o}\left(\mathrm{e}^{\frac{\frac{V}{N_{s}}+\frac{I}{N_{p}} R_{s}}{n V_{T}}}-1\right)-N_{p} \frac{\frac{V}{N_{s}}+\frac{I}{N_{p}} R_{s}}{R_{s h}},
$$

where $N_{s}$ and $N_{p}$ are the numbers of series and parallel connected cells, respectively.

\subsection{Extracting the SDM five parameters}

To determine the SDM five parameters, the implicit form of the SDM shown in Eq. (1) has to be solved. The implicit transcendental nature of this equation is based on the fact that neither the cell current nor its voltage can be explicitly expressed as a function of elementary terms [14]. Since it is only one equation with five unknowns, therefore, a computer intelligence method is required to calculate these unknowns; like optimization methods or a genetic algorithm. This paper uses the particle swarm optimization algorithm to solve this equation and determine the five parameters.

PSO is one of the well-regarded algorithms in the literature for optimization tasks and is widely used in both science and industry. PSO is well described in $[15,16]$; however, this section provides a short description of this algorithm. PSO works on a population of potential solutions (particles) within a search space (5-dimensional in this paper) by following the current optimum particles. PSO follows three rules: each particle needs to record its best solution achieved so far, all swarm particles need to communicate and update the global best solution reached and each particle needs to note the next movement speed and direction (velocity). The velocity of each particle is calculated as the tendency resulting from its current velocity, its best solution (local best), and all swarm best solution (global best). The formal expression for calculating the particle next velocity is given as:

$$
\mathbf{V}_{i}^{t+1}=w \mathbf{V}_{i}^{t}+c_{1} r_{1}\left(\mathbf{P}_{i}^{t}-\mathbf{X}_{i}^{t}\right)+c_{2} r_{2}\left(\mathbf{G}^{t}-\mathbf{X}_{i}^{t}\right),
$$

where $i$ is the index of the particle; $t$ is the iteration number; $\mathbf{V}_{i}^{t+1}$ is the particle next velocity; $\mathbf{V}_{i}^{t}$ is the current particle velocity; $\mathbf{X}_{i}^{t}$ is the current particle position; $\mathbf{P}_{i}^{t}$ is the particle local best; $\mathbf{G}^{t}$ is the global best; $w$ is the inertia weight that tunes between exploration and exploitation, $c_{1}$ and $c_{2}$ are control parameters and $r_{1}$ and $r_{2}$ are random values between 1 and 0 . It is worth mentioning here that $w$ is normally decreased linearly (usually: $1>w>0$ ) with increasing $t$, to gradually change from exploration to exploitation. $c_{1}$ and $c_{2}$ are used to balance between the particle individual and social tendency (last two terms in Eq. (3)). $r_{1}$ and $r_{2}$ are for indicating the stochastic nature of the PSO by randomly changing the weights of the individual and the social components. The particle next position is calculated using the following expression:

$$
\mathbf{X}_{i}^{t+1}=\mathbf{X}_{i}^{t}+\mathbf{V}_{i}^{t+1}
$$

\subsection{Constructing the electrical sub-model}

The electrical sub-model is built using a method presented in previous research [17]. The proposed method is based on dividing the voltage axis into a fixed interval and calculating the current values at a wide range of environmental conditions using the extracted five parameters by substituting the extracted parameters into the Lambert $\mathrm{W}$ function-based explicit form of the SDM, which is given as $[18,19]$ :

$$
\begin{aligned}
I= & \frac{R_{s h}\left(I_{p h}+I_{o}\right)}{R_{s h}+R_{s}}-\frac{n V_{T}}{R_{s}} W\left(\frac{R_{s} I_{o}}{n V_{T}} \frac{R_{s h}}{R_{s h}+R_{s}} e^{\left.\frac{R_{s h}\left(V+R_{s h}\left(I_{p h}+I_{o}\right)\right)}{n V_{T}\left(R_{s h}+R_{s}\right)}\right)}\right. \\
& -\frac{V}{R_{s h}+R_{s}},
\end{aligned}
$$

where $W$ is the principal branch of the Lambert $\mathrm{W}$-function. 
Considering the availability of measurement at different temperatures and irradiance conditions, a two-dimensional matrix of current values will be calculated for each voltage interval point. A polynomial surface function is required to be fitted to the current matrix data to generate a sub-model corresponding to each voltage interval point. For this purpose, a fifth-order polynomial surface function has been used. Hence, there are 21 coefficients for each voltage interval points. Although there is a large number of coefficients; however, calculating the coefficients has to be done only once.

The mathematical representation of the sub-models resulting from the fitted function is given as:

$$
\begin{aligned}
I_{\text {out }}^{i}= & \sum_{k=1}^{3} B_{k}^{i} \cdot T^{k-1} \cdot G^{k-1}+\sum_{l=2}^{4} B_{l+2}^{i} \cdot T \cdot G^{l} \\
& +\sum_{m=2}^{4} B_{m+5}^{i} \cdot T^{m} \cdot G+\sum_{n=1}^{5} B_{n+9}^{i} \cdot T^{n}+\sum_{p=1}^{5} B_{p+14}^{i} \cdot G^{p} \\
& +\sum_{q=2}^{3} B_{q+18}^{i} \cdot T^{5-q} \cdot G^{q}
\end{aligned}
$$

where $I_{\text {out }}^{i}$ is the calculated current value corresponding to a specific voltage interval point $i ; B_{i}^{1}-B_{i}^{21}$ are the fitting coefficients corresponding to the surface function of the current at the same voltage interval point depending on the irradiation and the temperature. $T$ and $G$ are the targeted temperature and irradiance conditions, respectively, at which a new $I-V$ curve is required to be constructed.

The final step is to calculate the current corresponding to each voltage interval point using the corresponding coefficients as a function of the targeted $T$ and $G$, and by considering only the $I-V$ points in the first power quadrant. After this the generate power can be selected as the $I-V$ product with the highest value.

\section{THERMAL SUB-MODEL}

The PV energy balance can be represented as the difference between the incident energy and the electrically generated energy [20]. The heat energy can be exchanged between the top and bottom surfaces of the PV module and the surrounding media via three mechanisms: conduction, convection, and radiation [21, 22]. The heat conduction mechanism can be neglected in normal situations since the frame structure that holds the PV module is in touch only with the thin sidewalls of the PV module, which has relatively small area. Also, the thermal exchange through side surfaces is neglected because of its small area compared to the top and the bottom surfaces.

The rest of this section is dedicated for discussing steps of implementing the thermal sub-model:

Input data from multiple sources are required:

- environmental measurements data;

- result of the Bird's clear sky model (mainly the solar irradiance);
- PV module technological and material parameters and properties (such as thermal conductivity, kinematic viscosity, Prandtl number and cell efficiency);

- and structural parameters of the PV cell that define its dimensions.

The module physical parameters values at the current temperature are calculated using a linear relationship based on their reference values.

Calculating the heat transfer coefficients for both free and forced convection from both top and bottom surfaces given as [23]:

$$
\begin{gathered}
h_{\text {forced }}=\frac{k}{L}\left(0.0308 \cdot \operatorname{Re}^{0.8} \cdot \operatorname{Pr}^{0.33}\right), \\
h_{\text {free_t }}=\frac{k}{L C}\left(0.54 \cdot\left(\frac{g \cdot \beta \cdot\left(T+\Delta T_{t}-T_{a}\right) \cdot L C^{3} P r}{n i^{2}}\right)^{0.25}\right), \\
h_{\text {free_b }}=\frac{k}{L C}\left(0.27 \cdot\left(\frac{g \cdot \beta \cdot\left(T+\Delta T_{b}-T_{a k}\right) \cdot L C^{3} \cdot P r}{n i^{2}}\right)^{0.25}\right),
\end{gathered}
$$

The forced convection coefficient $\left(h_{\text {forced }}\right)$ is considered to be the same for both top and bottom surfaces, $h_{\text {free_t }}$ and $h_{\text {free_b }}$ are the free convection coefficients of the top and bottom surfaces respectively. In the above three equations the following parameters have been used: $k$ is the thermal conductivity; $L$ is the length of the PV sample; $L C$ is the characteristic length, which equals to $L \cdot W / 2(L+W) ; W$ is the width; Re is Reynold's number; $P r$ is the Prandtl's number; $g$ is the standard gravity; $\beta$ is the thermal expansion coefficient and $\Delta T_{t}$ and $\Delta T_{b}$ are the temperature difference between the cell and the top and bottom surfaces respectively.

Calculating the heat flux for both convection mechanisms follow the general form shown below $[2,23]$ :

$$
\dot{Q}=h \cdot L \cdot W \cdot\left(T+\Delta T-T_{a k}\right),
$$

where $\dot{Q}$ refers to the heat flux.

Calculating the heat flux due to radiation from both surfaces using the following general expression:

$$
\dot{Q}=\sigma \cdot L \cdot W \cdot\left(T_{x}^{4}-T_{y}^{4}\right)
$$

where $T_{x}$ is the module surface temperature, $T_{y}$ is the sky or ground temperature and $\sigma$ is the universal Boltzmann's constant.

Calculate the resultant heat flux for both surfaces by summing the related components.

The balance heat energy concept is implemented using the following equation:

$$
\dot{Q}_{T}+\dot{Q}_{B}-P_{\text {dissp }}=0,
$$

where $\dot{Q}_{T}$ and $\dot{Q}_{B}$ are the total heat flux from the top and the bottom surfaces, respectively. $P_{\text {dissp }}$ is the total dissipated power given as: 


$$
P_{\text {dissp }}=G \cdot L \cdot W \cdot(1-\eta),
$$

where $G$ is the solar irradiance, and $\eta$ is the efficiency at the actual temperature.

At this point, the task is to minimize the error results from Eq. (12) and determine the correct values of $T, \Delta T_{t}$, and $\Delta T_{b}$ by using an iterative method. For this purpose, Newton-Raphson iteration has been selected as a solving method using MAPLE software environment.

\section{RESULTS AND DISCUSSION}

Validating the proposed model was carried out using a wide range of measurements for a mono-crystalline solar cell. Quantifying the error and evaluating the performance of the electrical sub-model is implemented by calculating the error at the maximum power point $(\delta)$, the coefficient of determination $\left(R^{2}\right)$, the Mean Absolute Percentage Error (MAPE) and the Algorithm Convergence Time (ACT). Collecting the required measurement was performed during previous research in which the irradiance range was $200-1,200 \mathrm{~W} / \mathrm{m}^{2}$ and the temperature range were $10-85^{\circ} \mathrm{C}$, under laboratory environments [18]. Figs 1 and 2 show the effectiveness of the

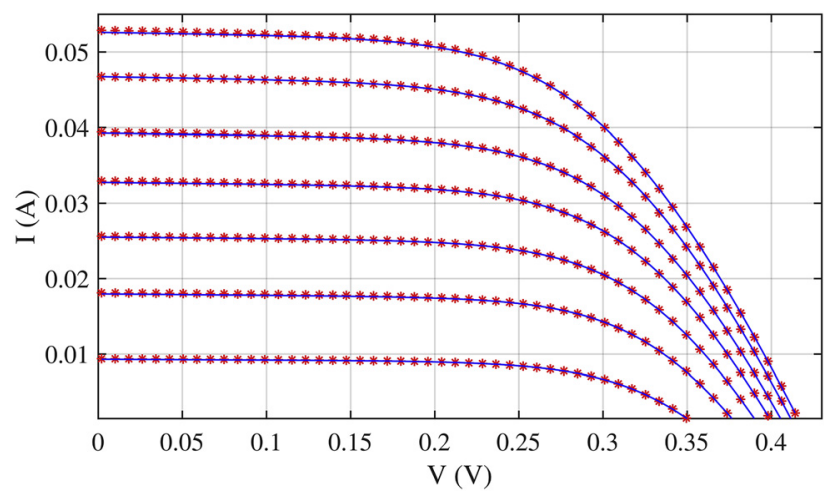

Fig. 1. SDM implicit form - PSO method applied for a monocrystalline solar cell, curves of different irradiance conditions

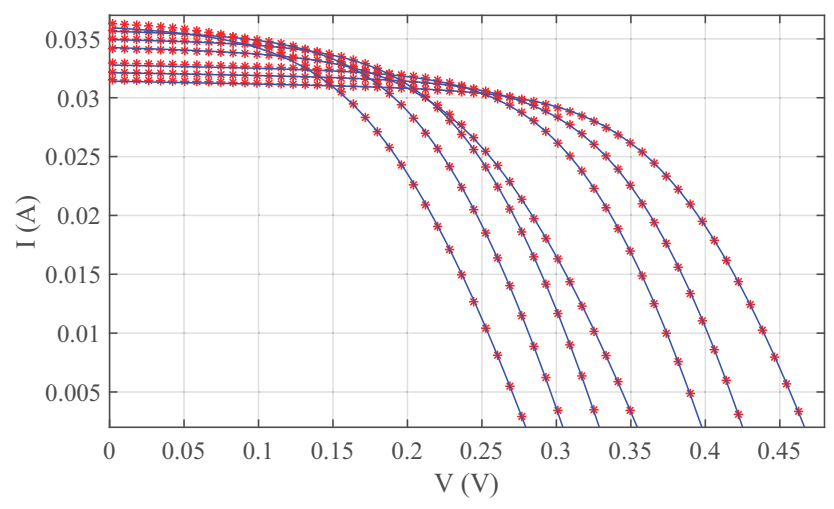

Fig. 2. SDM implicit form - PSO method applied for a monocrystalline solar cell, curves of different temperatures parameters extraction method using PSO under the aforementioned irradiances and temperatures, respectively.

Figs 1 and 2 show the applicability of the proposed method for extracting the SDM five parameters. Fig. 1 results from substituting the extracted parameters for $I-V$ curves measured at the same temperature but different irradiance in the Lambert $\mathrm{W}$ function and plot them together with the measured $I-V$ curves. Fig. 2 deal with the same concept but this time the $I-V$ curves measured at the same irradiance but different temperatures.

Table 1 shows how the extracted parameters are related to their physical expected values. Moreover, the result reflects some physical characteristics of the solar cells.

Table 2 shows the performance monitoring parameters of the electrical sub-model when extracting the characteristics parameters shown previously in Table 1.

The results of reproducing the $I-V$ curves using the extracted parameters at the corresponding environmental condition are shown together with the measured data in Figs 3 and 4 . Evaluating the electrical sub-model has been performed in Table 3 using $456 I-V$ curves measured at a

Table 1. Electrical sub-model performance monitoring parameters

\begin{tabular}{lcccccl}
\hline$G\left(\mathrm{~W} / \mathrm{m}^{2}\right)$ & $T\left({ }^{\circ} \mathrm{C}\right)$ & $I_{p h}(\mathrm{~A})$ & $I_{o}(\mathrm{~A})$ & $R_{s h}(\Omega)$ & $R_{s}(\Omega)$ & $n$ \\
\hline 200 & 25 & 0.0090 & $1.8 \cdot 10^{-7}$ & 900 & 1.74 & 1.48 \\
400 & & 0.0174 & $3.2 \cdot 10^{-7}$ & 582.8 & 1.38 & 1.55 \\
600 & & 0.0246 & $2.3 \cdot 10^{-7}$ & 460.7 & 1.7 & 1.54 \\
800 & & 0.0316 & $2.8 \cdot 10^{-7}$ & 428.3 & 1.66 & 1.5 \\
1,000 & & 0.0378 & $2.2 \cdot 10^{-7}$ & 376.7 & 1.73 & 1.52 \\
1,118 & & 0.0451 & $1.7 \cdot 10^{-7}$ & 300 & 1.62 & 1.46 \\
1,268 & & 0.0510 & $4.3 \cdot 10^{-7}$ & 299.4 & 1.67 & 1.6 \\
800 & 10 & 0.0315 & $6.1 \cdot 10^{-8}$ & 337.4 & 1.73 & 1.49 \\
& 20 & 0.0317 & $2.1 \cdot 10^{-7}$ & 428.3 & 1.66 & 1.5 \\
& 30 & 0.0322 & $6.1 \cdot 10^{-7}$ & 465.4 & 1.36 & 1.54 \\
& 40 & 0.0334 & $8.5 \cdot 10^{-7}$ & 403.8 & 2.27 & 1.36 \\
& 50 & 0.0339 & $6.0 \cdot 10^{-7}$ & 347.3 & 2.9 & 1.22 \\
& 60 & 0.0357 & $5.4 \cdot 10^{-6}$ & 316.2 & 1.73 & 1.21 \\
& 70 & 0.0361 & $1.1 \cdot 10^{-5}$ & 311.4 & 1.66 & 1.2 \\
\hline
\end{tabular}

Table 2. Results of the parameter extraction proposed method of different irradiance conditions and different temperatures

\begin{tabular}{lccccc}
\hline$G\left(\mathrm{~W} / \mathrm{m}^{2}\right)$ & $T\left({ }^{\circ} \mathrm{C}\right)$ & $\delta$ & $R^{2}$ & MAPE\% & ACT $(\mathrm{s})$ \\
\hline 200 & 25 & 0.053 & 0.99991 & 0.096 & 3.32 \\
400 & & 0.088 & 0.99997 & 0.047 & 3.36 \\
600 & & 0.067 & 0.99998 & 0.071 & 3.31 \\
800 & & 0.004 & 0.99991 & 0.073 & 3.36 \\
1,000 & & 0.133 & 0.99994 & 0.042 & 3.34 \\
1,118 & & 0.14 & 0.99985 & 0.055 & 3.31 \\
1,268 & & 0.071 & 0.99998 & 0.061 & 3.39 \\
800 & 10 & 0.294 & 0.99978 & 0.057 & 3.39 \\
& 20 & 0.004 & 0.99991 & 0.074 & 3.36 \\
& 30 & 0.159 & 0.99997 & 0.012 & 3.12 \\
& 40 & 0.079 & 0.99999 & 0.02 & 3.25 \\
& 50 & 0.054 & 0.99982 & 0.07 & 3.2 \\
& 60 & 0.068 & 0.99997 & 0.068 & 3.15 \\
& 70 & 0.008 & 0.99972 & 0.085 & 3.12 \\
\hline
\end{tabular}




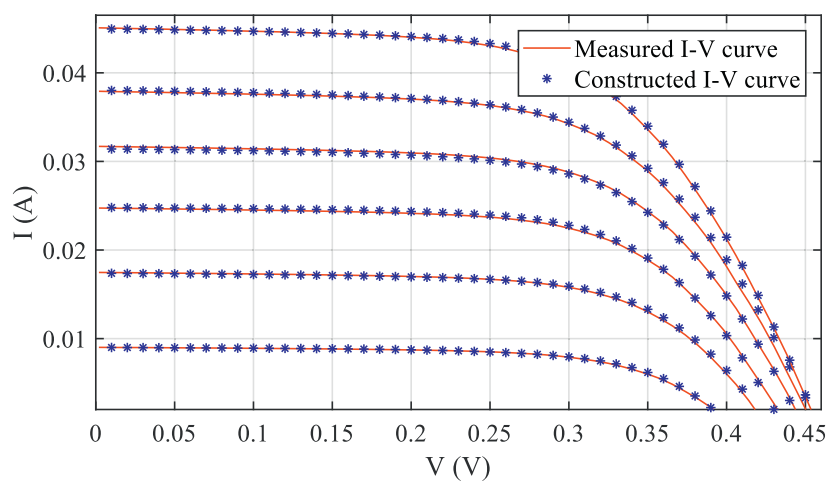

Fig. 3. New constructed I-V curves using the proposed electrical sub-model together with the measured data; at $1,000 \mathrm{~W} / \mathrm{m}^{2}$ and different temperatures $(10-85){ }^{\circ} \mathrm{C}$

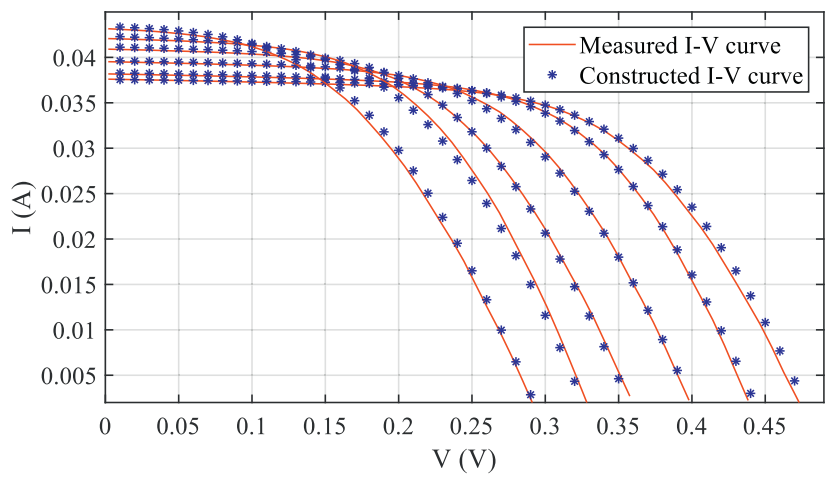

Fig. 4. New constructed I-V curves using the proposed electrical sub-model together with the measured data at $20^{\circ} \mathrm{C}$ and different irradiance conditions $(200-1,200) \mathrm{W} / \mathrm{m}^{2}$

Table 3. Evaluation of the proposed electrical sub-model using 456 curves measured at different environmental conditions

\begin{tabular}{lccc}
\hline Parameter & $\begin{array}{c}\text { Average } \\
\text { value }\end{array}$ & $\begin{array}{c}\text { Worst } \\
\text { recorded value }\end{array}$ & $\begin{array}{c}\text { Percentage of curves } \\
\text { with error value }>5 \%\end{array}$ \\
\hline$\delta$ & $2.11 \%$ & $5.69 \%$ & $2.10 \%$ \\
MAPE & $1.67 \%$ & $4.91 \%$ & $0.00 \%$ \\
\hline
\end{tabular}

wide range of irradiance conditions and temperatures, as mentioned previously.

The presented results validate the presented sub-models and show its applicability over a wide range of irradiance conditions and temperatures. The rest of this section the main insights of reviewing the collected results are listed.

The adopted electrical sub-model, which is based on the extracted SDM five parameters is able to reproduce a complete $I-V$ characteristic at any given temperature and irradiance. However, for building this model, it requires a full measured $I-V$ curve at a wide range of environmental conditions. To validate the electrical sub-model, Figs 3 and 4 show the reproduced $I-V$ curve at different irradiance conditions and temperatures.

Table 3 summarizes the electrical sub-model for 456 $I-V$ curves. The listed results show that the average value of the MAPE $1.67 \%$ which describes how close the reproduced $I-V$ curve and the measured one are at the same environmental conditions. It can be seen that the error at the maximum power point $(\delta)$ is about $2.11 \%$, which is an important parameter for accurate power prediction. Although the worst recorded $\delta$ was about $5 \%$, however, only $2.1 \%$ out of the 456 curves have values higher than $5 \%$.

The mathematical implementation of the thermal submodel has shown convergence in all cases. The verification of the thermal sub-model has been done by changing the incident irradiance over a wide range and running the model to calculate the resulting cell temperature and the temperature difference to the top and bottom surfaces. Table 4 (columns 1-4) shows the result of running the thermal submodel at $26{ }^{\circ} \mathrm{C}$ ambient temperature.

Table 4 (columns 5-8) shows the result of running the thermal sub-model at $800 \mathrm{~W} / \mathrm{m}^{2}$ of incident irradiance and different ambient temperatures.

From the above table a direct dependence of the cell temperate on the environmental conditions, namely temperature and irradiance can be realized. The resulting cell temperature, as expected, is much higher than the ambient temperature due to the fact that the bigger portion of the incident irradiance is converted to heat. It is also clear from the above results that the temperature difference between the cell and the top surface is higher than the difference to the

Table 4. Thermal sub-model results corresponding to different ambient temperatures and irradiances

\begin{tabular}{|c|c|c|c|c|c|c|c|}
\hline \multicolumn{4}{|c|}{ Constant ambient temperature $\left(26^{\circ} \mathrm{C}\right)$} & \multicolumn{4}{|c|}{ Constant irradiance $\left(800 \mathrm{~W} / \mathrm{m}^{2}\right)$} \\
\hline Irrad. $\left[\mathrm{W} / \mathrm{m}^{2}\right]$ & Cell Temp. $\left[{ }^{\circ} \mathrm{C}\right]$ & $\Delta T_{t}\left[{ }^{\circ} \mathrm{C}\right]$ & $\Delta T_{b}\left[{ }^{\circ} \mathrm{C}\right]$ & Ambient Temp. $\left[{ }^{\circ} \mathrm{C}\right]$ & Cell Temp. $\left[{ }^{\circ} \mathrm{C}\right]$ & $\Delta T_{t}\left[{ }^{\circ} \mathrm{C}\right]$ & $\Delta T_{b}\left[{ }^{\circ} \mathrm{C}\right]$ \\
\hline 200 & 29.19 & -0.07 & -0.08 & 0 & 24.71 & -0.22 & -0.54 \\
\hline 300 & 32.76 & -0.10 & -0.16 & 5 & 29.55 & -0.22 & -0.54 \\
\hline 400 & 36.29 & -0.12 & -0.24 & 10 & 34.40 & -0.23 & -0.54 \\
\hline 500 & 39.79 & -0.15 & -0.32 & 15 & 39.26 & -0.23 & -0.55 \\
\hline 600 & 43.24 & -0.17 & -0.40 & 20 & 44.14 & -0.23 & -0.55 \\
\hline 700 & 46.66 & -0.20 & -0.48 & 25 & 49.04 & -0.23 & -0.56 \\
\hline 800 & 50.03 & -0.23 & -0.56 & 30 & 53.97 & -0.23 & -0.57 \\
\hline 900 & 53.37 & -0.25 & -0.64 & 35 & 58.92 & -0.23 & -0.58 \\
\hline 1,000 & 56.67 & -0.29 & -0.73 & 40 & 63.90 & -0.23 & -0.58 \\
\hline
\end{tabular}


bottom surface because the heat transfer mechanisms are more effective in the upper surface where the radiation heat transfer is higher because the difference between the cell temperature and the sky temperature is higher than the difference to the ambient temperature, which is considered in the case of calculating the radiation from the bottom surface.

\section{CONCLUSION}

This paper proposes a novel combined model for precise power prediction of a PV system depending on the measured data. Both electrical and thermal sub-models require an iterative numerical method for solving nonlinear equations, where convergence and finding excellent solutions were achieved in all verification levels.

The results show the applicability of the proposed method at a wide range of environmental conditions. Different error monitoring parameters were used to monitor the applicability of the adopted methods. The rest of this section will summarize the main concluded concepts.

The electrical sub-model is build based on measurement data; that is, it is not restricted to the type of PV technology or other physical parameters. Hence, it is applicable for any type of PV module, as long as the PV module can be described by the single diode model.

A limitation of using the electrical sub-model is defined by the need for $\mathrm{I}-\mathrm{V}$ measurement under a wide range of environmental conditions.

The electrical sub-model has the advantage of simple implementation as well as the high accuracy results as discussed in Section 4.

The proposed thermal sub-model provides not only the cell temperature but also the temperature difference to both top and back surfaces. This data will be useful in predicting the temperature profile across the PV module. Hence, it helps to investigate the mechanical properties of the module and make conclusions about reliability related issues.

From the results shown in Section 4, it can be seen that the temperature differences between the junction and both surfaces are small. This is due to the small thermal resistivity of the PV mono-crystalline cell used to verify this model. This module is fabricated for research purposes, as discussed in a previous research work.

The proposed thermal model considers the effect of all heat transfer mechanisms from both module top and back surfaces, aiming for higher accuracy. Although it uses Newton-Raphson method as a solving algorithm, the model complexity is still in a very acceptable range. Evaluating and displaying the results is achievable in a matter of seconds, using a MATLAB environment installed on a computer with normal specifications.

\section{ACKNOWLEDGMENTS}

The research reported in this paper was supported by the BME Nanotechnology and Materials Science TKP2020 IE grant of NKFIH Hungary (BME IE-NAT TKP2020), the Stipendium Hungaricum Scholarship Programme, the grant EFOP-3.6.1-16-2016-00014 and by the Science Excellence Program at BME under the grant agreement NKFIH-849-8/ 2019 of the Hungarian National Research, Development and Innovation Office.

\section{REFERENCES}

[1] S. Shapoval, V. Zhelykh, N. Spodyniuk, O. Dzeryn, and B. Gulai, "The effectiveness to use the distribution manifold in the construction of the solar wall for the conditions of circulation," Pollack Period., vol. 14, no. 2, pp. 143-154, 2019.

[2] S. Armstrong and W. G. Hurley, "A thermal model for photovoltaic panels under varying atmospheric conditions," Appl. Therm. Eng., vol. 30, no. 11-12, pp. 1488-1495, 2010.

[3] E. Skoplaki and J. A. Palyvos, "Operating temperature of photovoltaic modules: A survey of pertinent correlations," Renew. Energ., vol. 34, no. 1, pp. 23-29, 2009.

[4] E. Skoplaki and J. A. Palyvos, "On the temperature dependence of photovoltaic module electrical performance: A review of efficiency/ power correlations," Solar Energy, vol. 83, no. 5, pp. 614-624, 2009.

[5] I. Haber and I. Farkas, "Analysis of the air-flow at photovoltaic modules for cooling purposes," Pollack Period., vol. 7, no. 1, pp. 113-121, 2012.

[6] R. S. Balog, Y. Kuai, and G. Uhrhan, "A photovoltaic module thermal model using observed insolation and meteorological data to support a long life, highly reliable module-integrated inverter design by predicting expected operating temperature," in IEEE Congress and Exposition on Energy Conversion, San Jose, CA, USA, Sep. 20-24, 2009, 2009, pp. 3343-3349.

[7] P. Guerriero, L. Codecasa, V. D'Alessandro, and S. Daliento, "Dynamic electro-thermal modeling of solar cells and modules," Solar Energy, vol. 179, pp. 326-334, 2019.

[8] W. Gu, T. Ma, L. Shen, M. Li, Y. Zhang, and W. Zhang, "Coupled electrical-thermal modeling of photovoltaic modules under dynamic conditions," Energy, vol. 188, Paper no. 116043, 2019.

[9] B. Plesz, Zs. Kohári, P. G. Szabó, A. Timár, and G. Bognár, "Novel semi-empirical combined electro-thermal model for solar modules," in 31st European Photovoltaic Solar Energy Conference and Exhibition, Münich, Germany, Sept. 14-18, 2015, 2015, pp. 2014-2018.

[10] M. U. Siddiqui and A. F. M. Arif, "Electrical, thermal and structural performance of a cooled PV module: Transient analysis using a multiphysics model," Appl. Energ., vol. 112, pp. 300-312, 2013.

[11] A. K. Abdulrazzaq, G. Bognár, and B. Plesz, "Evaluation of different methods for solar cells/modules parameters extraction," Solar Energy, vol. 196, pp. 183-195, 2020.

[12] M. Chegaar, Z. Ouennoughi, and A. Hoffmann, "A new method for evaluating illuminated solar cell parameters," Solid-State Electron., vol. 45, no. 2, pp. 293-296, 2001.

[13] T. Ma, H. Yang, and L. Lu, "Solar photovoltaic system modeling and performance prediction," Renew. Sustain. Energ. Rev., vol. 36, pp. 304-315, 2014.

[14] X. Gao, Y. Cui, J. Hu, G. Xu, and Y. Yu, "Lambert W-function based exact representation for double diode model of solar cells: 
Comparison on fitness and parameter extraction," Energ. Convers. Manage., vol. 127, pp. 443-460, 2016.

[15] Y. Shi and R. C. Eberhart, "Parameter selection in particle swarm optimization," in Evolutionary Programming VII, V. W. Porto, N. Saravanan, D. Waagen, and A. E. Eiben, Eds. Lecture Notes in Computer Science, vol. 1447. Springer, pp. 591-600, 1998.

[16] B. Jarboui, N. Damak, P. Siarry, and A. Rebai, "A combinatorial particle swarm optimization for solving multi-mode resourceconstrained project scheduling problems," Appl. Maths. Comput., vol. 195, no. 1, pp. 299-308, 2008.

[17] A. K. Abdulrazzaq, G. Bognár, and B. Plesz, "An efficient and simple method for modeling solar cells," Period. Polytech. Electr. Eng. Comput. Sci., vol. 64, no. 1, pp. 40-46, 2019.

[18] B. Plesz and J. Mizsei, "Influence of the photoactive layer thickness on the device parameters and their temperature dependence in thin crystalline silicon photovoltaic devices," in 22nd International
Workshop on Thermal Investigations of ICs and Systems, Budapest, Hungary, Sep. 21-23, 2016, 2016, pp. 311-314.

[19] A. K. Abdulrazzaq, "Electro-thermal modeling of photovoltaic (PV) systems," in Conference on Spring Wind, Györ, Hungary, vol. III, May 4-6, 2018, 2018, pp. 17-29.

[20] G. Notton, C. Cristofari, M. Mattei, and P. Poggi, "Modeling of a double-glass photovoltaic module using finite differences," Appl. Therm. Eng., vol. 25, no. 17-18, pp. 2854-2877, 2005.

[21] O. Dupré, R. Vaillon, and M. A. Green, Thermal Behavior of Photovoltaic Devices. Springer, 2017.

[22] S. P. Aly, S. Ahzi, N. Barth, and A. Abdallah, "Using energy balance method to study the thermal behavior of PV panels under time-varying field conditions," Energ. Convers. Manage., vol. 175, pp. 246-262, 2018.

[23] Y. A. Çengel, J. M. Cimbala, and R. H. Turner, Fundamentals of Thermal-Fluid Science. McGraw-Hill, 2001. 